\title{
Manufacturing of mechanical elements with properties of metamaterials using 3D printing technology
}

\author{
Michat Braszkiewicz ${ }^{*}$ \\ Faculty of Mechanical Engineering and Robotics, AGH University of Science and Technology, \\ Cracow, Poland
}

\begin{abstract}
Metamaterials are materials engineered to have properties that are not found in naturally occurring materials, which opens a wide range of opportunities for creation of materials with completely new functionalities. Auxetics which are a type of metamaterials are unique because they are engineered to have a negative Poisson's ratio whereas naturally occurring materials have a positive one. Auxetics have shown some really promising energy absorption properties which can be widely applicable in e.g.: automotive (crash absorbers, suspension parts), medicine (prosthetics), clothing (shoe soles). Moreover, they are showing other properties superior to conventional materials such as: increased shear modulus better acoustic behaviour improved fracture toughness and other. Methods of modelling in CATIA V5 software and various manufacturing methods with the use of 3D printing technologies like MSLA (Masked Stereolithography Apparatus), Selective Laser Sintering (SLS) and Fused Deposition Modelling (FDM) are presented.
\end{abstract}

\section{Introduction}

Metamaterials are materials engineered to have properties that are not found in naturally occurring materials, which opens a wide range of opportunities for creation of materials with completely new, mind-blowing functionalities like creating an invisibility cloak.

Auxetic materials (mechanical metamaterials with a negative Poisson's ratio [1]) were first synthesised by Lakes in 1987 [2,3]. The term auxetics was first introduced by Evans in a paper published in Nature in 1991 from the root word auxesis which means growth in ancient Greek to describe expansion under tension $[4,5]$.

Auxetics have shown some really promising energy absorption properties [6,7] which can be widely applicable in e.g.: automotive (crash absorbers, suspension parts), medicine (prosthetics), clothing (shoe soles). Moreover, they are showing other properties superior to

* Corresponding author: braszkiewicz@agh.edu.pl 
conventional materials such as: increased shear modulus [8] better acoustic behaviour [9] improved fracture toughness [10] and other.

Since Lakes produced first isotropic PU foam many scientists are developing and testing similar structures like polymeric or metallic foams, microporous polymers, carbon fibre laminates and honeycomb structures [3]. In recent years due to progress in 3D printing and computer simulations development of those materials has rapidly accelerated.

\section{Modelling of metamaterial structures}

\subsection{Negative Poisson's ratio}

Auxetics are unique because they are engineered to have a negative Poisson's ratio which is defined as a negative ratio of lateral to linear strain.

$$
\text { Poisson's Ratio }=-\frac{\text { Lateral Strain }}{\text { Linear Strain }}
$$

where:

$$
\begin{aligned}
& \text { Lateral Strain }=\frac{\Delta d}{d_{0}}=\frac{d-d_{0}}{d_{0}} \\
& \text { Linear Strain }=\frac{\Delta l}{l_{0}}=\frac{l-l_{0}}{l_{0}}
\end{aligned}
$$

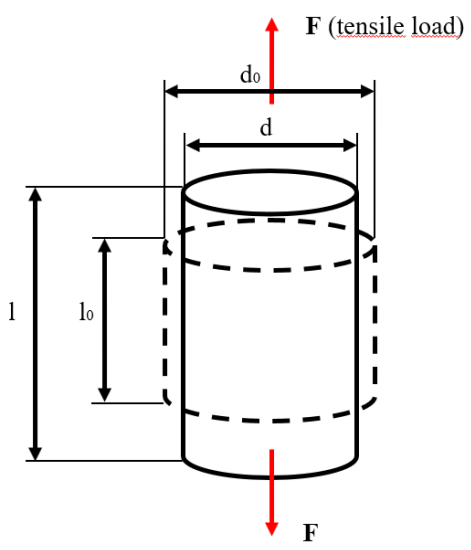

Fig. 1. Sample under load

Naturally occurring materials have a positive Poisson's ratio, because if a material is compressed or stretched then one of its dimensions decreases and other increases, which results in one of the strains value being negative and as a consequence the ratio being positive. Metamaterials can be structured in a way that both linear and lateral strain can be negative or positive at the same time (both perpendicular dimensions can increase or decrease at the same time) which leads to a negative Poisson's ratio [11]. To achieve this a retracting mechanism has to be embedded into the material. 


\subsection{Retracting mechanisms}

Retracting mechanisms can have different forms and shapes. Researchers are printing complex structures with different shapes of retracting mechanisms e.g. star shaped [12], triangular [13], double $\mathrm{V}$ [14], honeycomb [15]. A good demonstration of how such mechanism works is shown on fig. 2.
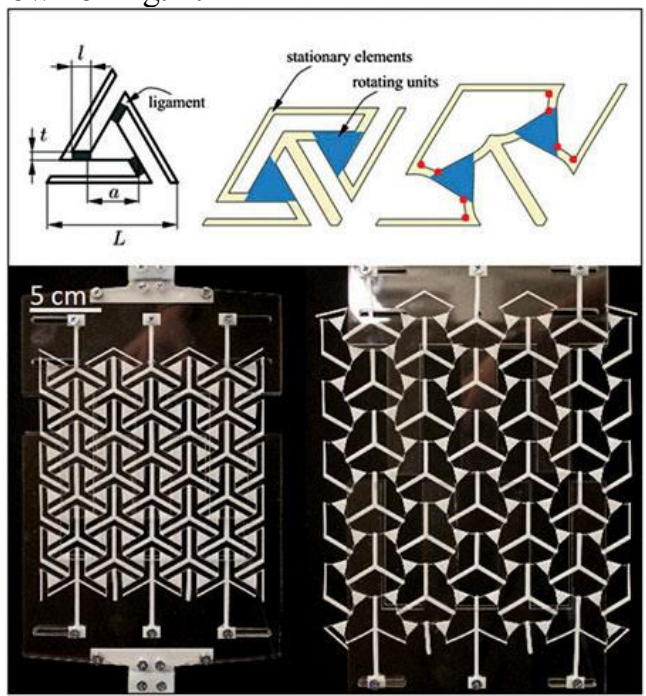

Fig. 2. Demonstration of how a retracting mechanism works [16]

Design of a metamaterial starts with the design of a retracting mechanism. The most important feature of the mechanism is to retract in such a manner, that the linear movement also enforces the lateral one in the same direction. This means that the mechanism has to retract or extract in both linear and lateral directions at the same time.

In this case a CATIA V5 software is used for design of the mechanism. The design is conducted in Generative Shape Design (GSD) and Part Design (PD) modules of the software. A view of a design of single retracting mechanism is shown in Fig.3.

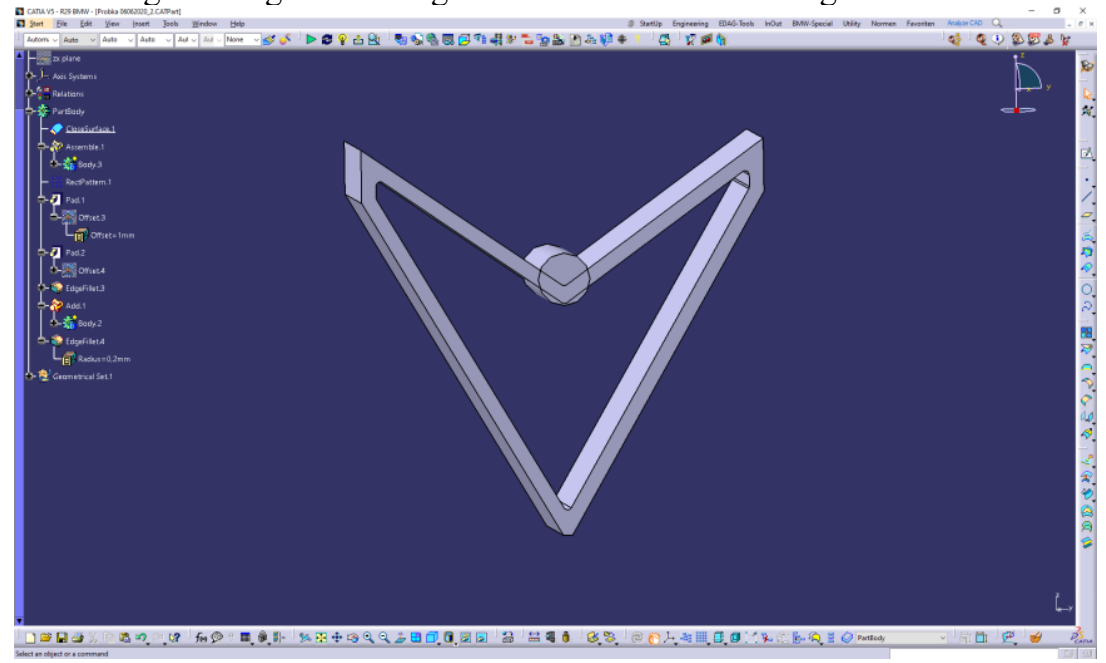

Fig. 3. Single retracting mechanism in CATIA V5 


\subsection{Modelling of a sample}

In order to create a sample ready for further investigation a retracting mechanism has to be multiplied with a use of a pattern in such a way that multiple joined mechanisms will be able to interact with each other resulting in a metamaterial of a negative Poisson's ratio. An example of such a material sample created in CATIA is shown in Fig.4.

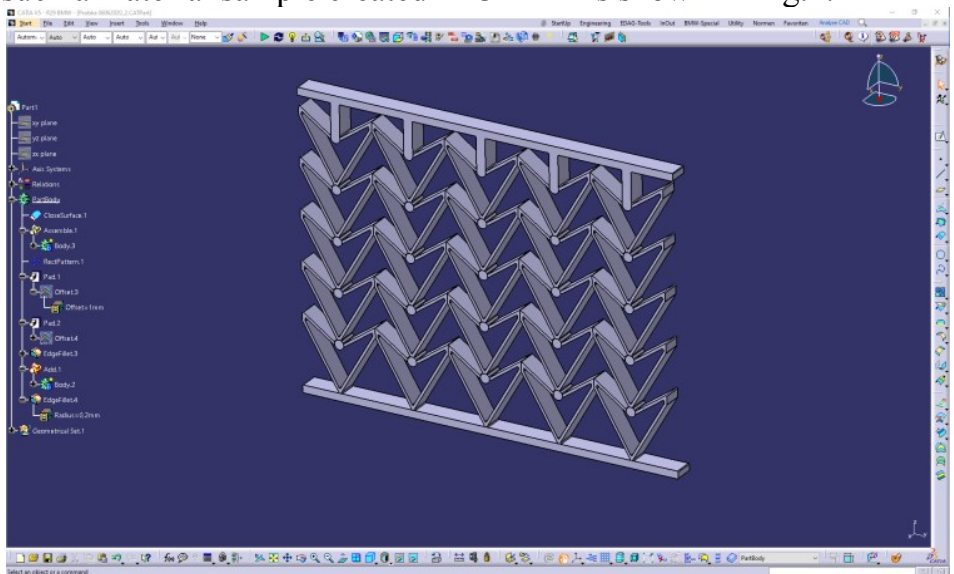

Fig. 4. Metamaterial sample in CATIA V5

\section{Manufacturing of metamaterials}

Since the first metamaterials were synthetised by Lakes in 1987 as simple foams much has changed in the process of manufacturing. Currently most research focuses on printing of metamaterial structures with the use of various $3 \mathrm{D}$ printing technologies.

\subsection{Fused Deposition Modelling (FDM)}

Fused Deposition Modelling (widely known as FDM) is the most popular method of 3D printing. In this method a plastic filament is heated in a nozzle which moves along $\mathrm{x}$ and $\mathrm{y}$ axis and then distributed among a heated bed. The bed lowers along $\mathrm{z}$ axis after every layer of new filament which results in a printed part consisting of multiple layers bonded with each other. Principle of FDM printed is shown in Fig. 5. This method is suitable only for printing very simple flat metamaterial structures such as shown in the Fig.6.

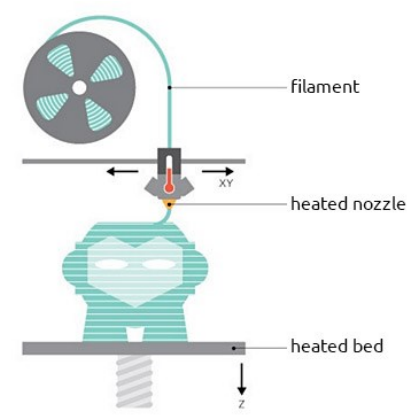

Fig. 5. Principle of FDM printing [17]

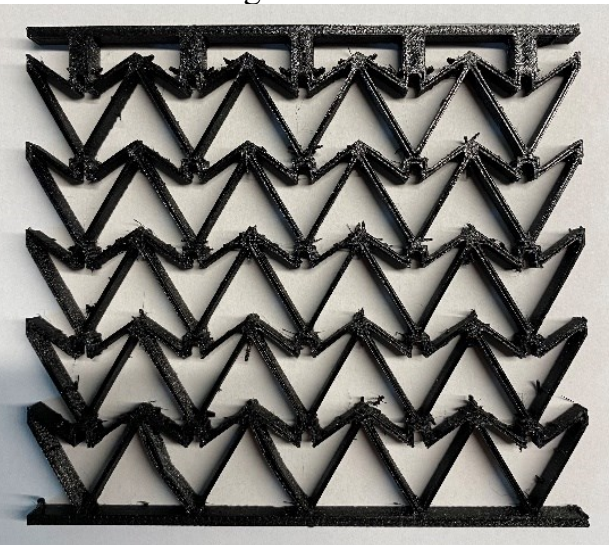

Fig. 6. Sample printed on a FDM printer 


\subsection{Selective Laser Sintering (SLS)}

Selective Laser Sintering (SLS) involves sintering polyamide or polystyrene powders. In this method the powder which layer lies on a printer bed is sintered with a laser beam. The bed is lowered after each layer and new powder stored in a container is spread among the print area with a roll.

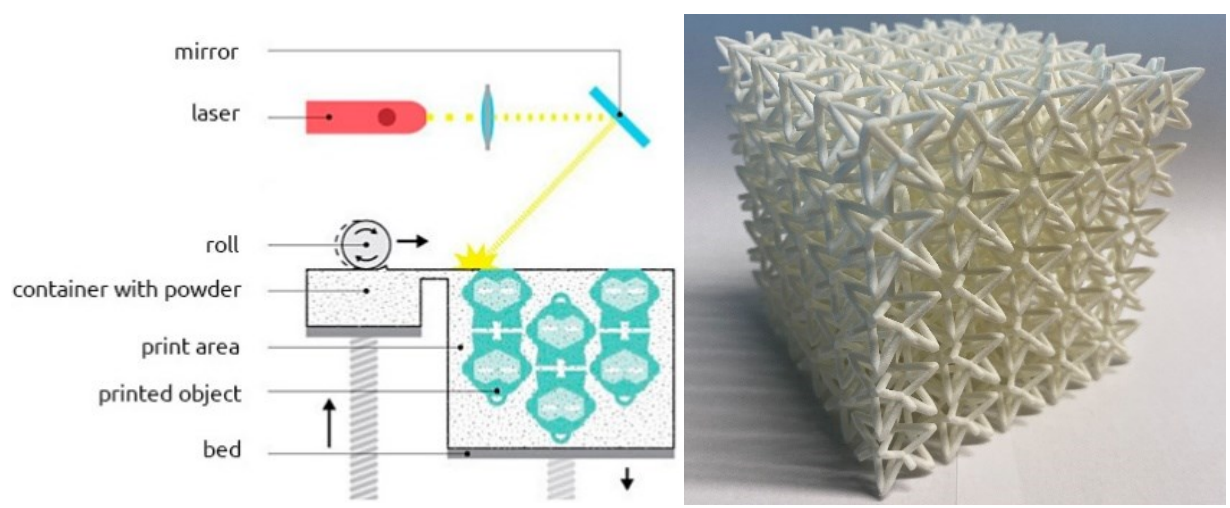

Fig. 7. Principle of SLS 3D printing [18]

Fig. 8. Sample printed with an SLS printed

Prints created with this technology are very strong and because the print is supported by the powder not much support is needed. The prints have a superb surface quality and dimensional accuracy. This method is well suited for structures with very complex geometries. A metamaterial structure manufactured with the use of this method is shown in Fig.8.

\subsection{Masked Stereolithography Apparatus (MSLA)}

Masked Stereolithography Apparatus (MSLA) is a method which uses a Photopolymer Resin which is curable in a $405 \mathrm{~nm}$ wavelength UV light. This resin is purred into a tank with a transparent bottom underneath which is an LCD screen. The screen displays light objects which have the shape of a particular layer of the print. After a layer is cured, the build platform is moved upwards along the $\mathrm{Z}$ axis of a thickness of a layer and then a next layer is cured. Layer after layer a finished object is created.

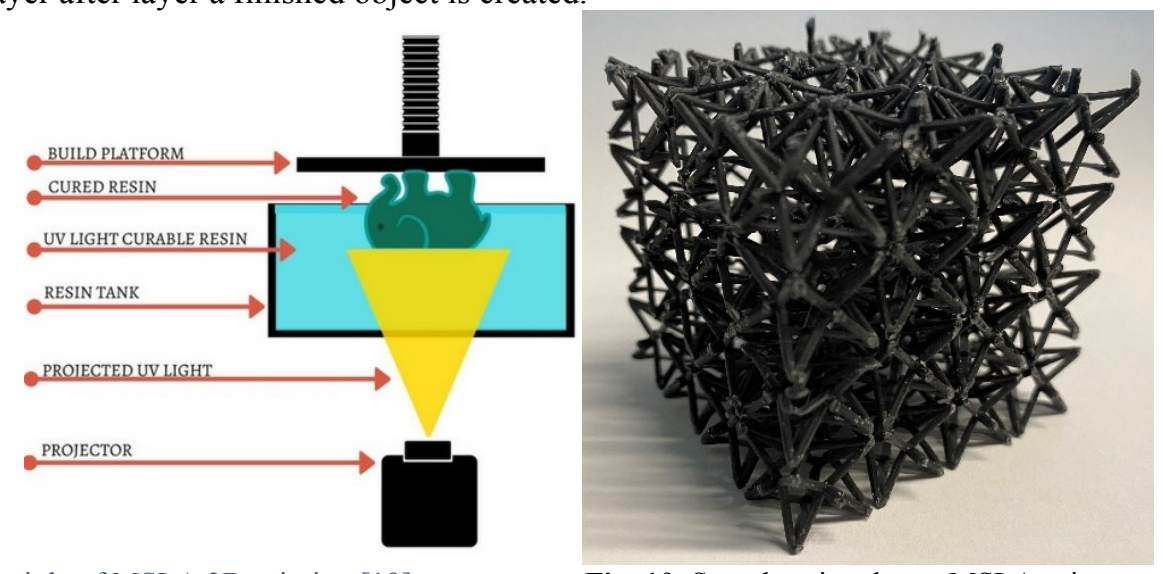

Fig. 9 Principle of MSLA 3D printing [19]

Fig. 10. Sample printed on a MSLA printer

An example of a metamaterial sample printed with the use of this method is presented in Fig. 10 . 


\subsection{Masked Stereolithography Apparatus (MSLA) with Monochrome Light}

The newest type of MSLA uses monochrome light or mono for short. Instead of using a colour LCD this technology uses LCD panels with just one wavelength which is more efficient and allows to drastically cut print times. To estimate the time savings in a print of a metamaterial sample an identical sample was printed both on a standard MSLA printer and a printer which uses mono light. The same slicer (Chitubox 1.7.0.) and Photoploymer Resin (Monocure 3D Flex) were used. The standard printer is Elegoo Mars which was compared to Elegoo Mars 2 Pro with mono light.

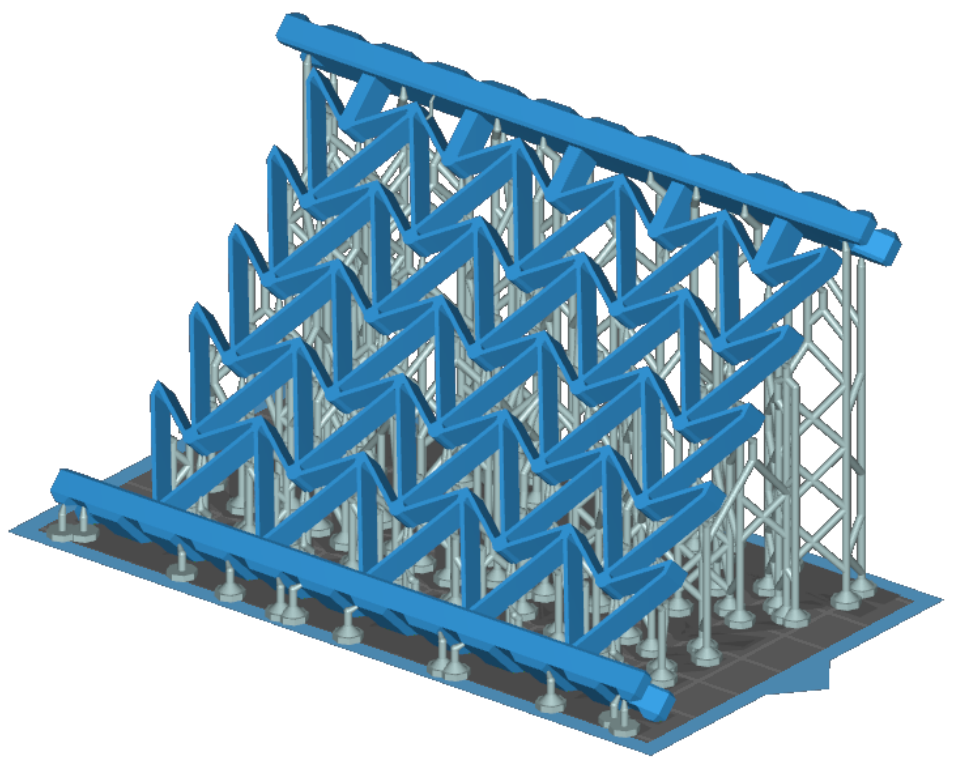

Fig. 11. Printed Sample with supports in slicer software Chitubox

Table 1. Printing parameters

\begin{tabular}{|c|c|c|}
\hline & Elegoo Mars & Elegoo Mars 2 Pro \\
\hline Layer Height & $0,05 \mathrm{~mm}$ & $0,05 \mathrm{~mm}$ \\
\hline Bottom Layer Count & 4 & 4 \\
\hline Exposure Time & $20 \mathrm{~s}$ & $10 \mathrm{~s}$ \\
\hline Bottom Exposure Time & $60 \mathrm{~s}$ & $60 \mathrm{~s}$ \\
\hline Light-off Delay & $1 \mathrm{~s}$ & $1 \mathrm{~s}$ \\
\hline Lifting Speed & $40 \mathrm{~mm} / \mathrm{min}$ & $40 \mathrm{~mm} / \mathrm{min}$ \\
\hline Retract Speed & $150 \mathrm{~mm} / \mathrm{min}$ & $210 \mathrm{~mm} / \mathrm{min}$ \\
\hline Print Time & $\mathbf{1 2 h} 4 \mathbf{m}$ & $\mathbf{0 8 h} 3 \mathbf{m}$ \\
\hline
\end{tabular}




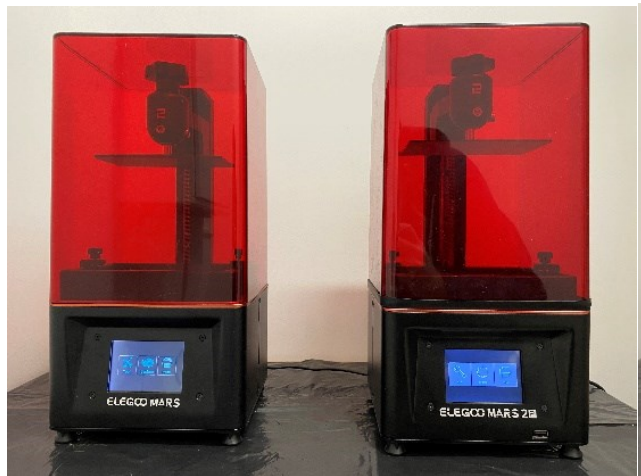

Fig. 12. Standard MSLA printer (Elegoo Mars) on the left and new MSLA printer with mono light (Elegoo Mars 2 Pro) on the right

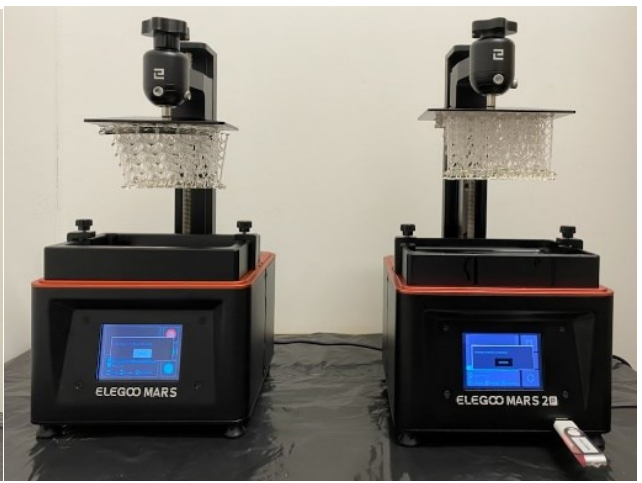

Fig. 13. Ready print on both printers

Mono light technology allowed in our case to cut printing time from $12 \mathrm{~h} 41 \mathrm{~m}$ for the Elegoo Mars with standard LCD to $08 \mathrm{~h} 34 \mathrm{~m}$ for the new Elegoo Mars 2 Pro. That means time savings of almost $35 \%$.

\section{References:}

[1] S. D. Poisson, Traité de Mécanique 2, 476 (1811)

[2] R. S. Lakes: Science, 1987, 235, 1038-1040.

[3] K. L. Alderson, A. Alderson, G. Smart, V. R. Simkins \& P. J. Davies Plastics, Rubber and Composites, 31:8, 344-349 (2002)

[4] Evans, K. E., Nkansah, M. A., Hutchinson, I. J. \& Rogers, S. C. Nature 353, 124, (1991)

[5] G. N. Greaves, A. L. Greer, R. S. Lakes, Nature Materials (2011)

[6] F. Scarpa, J.R. Yates, L.G. Ciffo, P I Mech. Eng. M-J Eng. 216 (12) 1153- 1156 (2002)

[7] W. Liu, N. Wang, T. Luo, Mater. Des. 100 84-91 (2016)

[8] K. E. Evans, A. Alderson, Adv. Mater. 12 (9) 617-628 (2000)

[9] R. S. Lakes, Science, 238551 (1987)

[10] P. Subramani, S. Rana, D.V. Oliveira, Mater. Des. 61 286-295 (2014)

[11] R. S. Lakes, Advanced Materials 5, 293-296 (1993).

[12]L. Mizzi, E.M. Mahdi, K. Titov, Materials and Design (Elsevier 2018)

[13] E. Andreassen, Boyan S. Lazarov, O. Sigmund Mechanics of Materials (Elsevier 2014)

[14] Y. Xue, F. Han Materials Letters (Elsevier 2019)

[15] D. Mousanezhad, et al. Sci. Rep. 5,18306 (2015)

[16] https://www.cambridge.org/core/journals/mrs-bulletin/news/kirigami-art-and-

geometric-manipulation-transform-rigid-solids-to-flexible-auxetic-materials

[17] https://b3d.com.pl/en/127/fdm-fused-deposition-modelling

[18] https://b3d.com.pl/31/sls-selective-laser-sintering

[19] https://pick3dprinter.com/dlp-3d-printing/

[20] P. Subramani, S. Rana, D.V. Oliveira, Mater. Des. 61 286-295 (2014) 
[21] T.-C Lim, Auxetic Materials and Structures. (Springer, 2015).

[22] R.S. Lakes \& R. Witt International Journal of Mechanical Engineering Education 30, 50-58 (2002)

[23] Yuanlong Wang, LiangmoWang, Zheng-dong Ma Materials and Design (Elsevier 2016)

[24] Hui Wang, Yuxuan Zhang, Wanqing Lin Computational Materials Science (Elsevier 2020)

[25] L. Francesconi, A. Baldi, X. Liang, Extreme Mechanics Letters (Elsevier 2019)

[26]Zhichao Dong, Ying Li, TianZhao, Materials and Design (Elsevier 2019)

[27] K. E. Evans: Chem. Ind., 1990, 20, 652-657 